\title{
Percepção subjetiva de memória e habilidade manual em idosos de uma oficina de inclusão digital
}

Subjective memory perception and manual ability among elderly from a workshop of digital inclusion

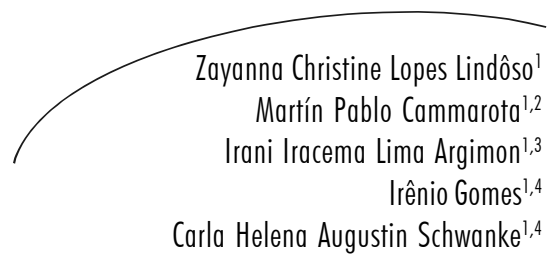

Resumo

Introdução: É notório que, para realizar satisfatoriamente as tarefas do cotidiano, é preciso destacar a importância da habilidade manual. A mão serve tanto como receptor de informações quanto executor de resposta. A habilidade manual interage juntamente com as funções cognitivas, formando um sistema complexo de movimentos integrados. Assim, o objetivo deste trabalho foi verificar a associação de percepção subjetiva de memória com a habilidade manual em idosos. Metodologia: foram avaliados 51 idosos entre 60 e 78 anos, participantes das Oficinas de Inclusão Digital do Projeto PotencialIdade do Instituto de Geriatria e Gerontologia da Pontifícia Universidade Católica do Rio Grande do Sul. Para o desenvolvimento da pesquisa, utilizou-se um questionário contendo dados sociodemográficos, a Avaliação Funcional Breve, o Teste de Percepção Subjetiva de Memória, os Testes de Memória Lógica I e II e o Action Research Arm Test (ARAT). Resultados: observou-se que as mulheres percebem sua memória de forma mais negativa que os homens. Houve correlação significativa entre a memória inicial e tardia, habilidade manual do lado direito com o lado esquerdo, habilidade manual do lado direito com a percepção subjetiva de memória. Conclusão: os resultados obtidos neste estudo reforçam a hipótese de que existe associação entre a percepção subjetiva de memória e habilidade manual. Contudo, estudos adicionais são necessários para aprofundar o conhecimento sobre a temática, uma vez que se trata de um estudo original.

\section{Abstract}

Introduction: In order to satisfactorily perform the tasks of daily life we must emphasize the importance of manual skills. The hand not only serves as a receiver of information, but also as an executer response. The manual skill interacts with

\footnotetext{
Pontifícia Universidade Católica do Rio Grande do Sul - PUCRS. Porto Alegre, RS, Brasil

Programa de Pós-graduação em Gerontologia Biomédica. PUCRS

2 Instituto do Cérebro, Laboratório de Neuroquimica \& Neurofisiologia da Memória/Centro de Memória. PUC-RS

3 Faculdade de Psicologia, Departamento de Psicologia Clínica. PUC-RS

4 Instituto de Geriatria e Gerontologia. PUC-RS

Correspondência / Correspondence

Zayanna Christine Lopes Lindôso

E-mail: zayanna@gmail.com
}

Palavras-chave: Cognição. Geriatra. Movimento. Percepção de memória. Habilidade manual. Inclusão digital. Idosos. Envelhecimento. 
the cognitive functions, forming a complex system of integrated movements. Thus, the objective of this work was to verify the association of subjective perception of memory with manual ability in elderly. Methodology: 51 elderly people between 60 and 78 years participating in the workshops of Digital Inclusion of the Potential/dade Project, of the Institute of Geriatrics and Geriatrics and Gerontology of the Pontifical Catholic University of Rio Grande do Sul were evaluated. For the development of the research, a questionnaire containing sociodemographic data, the Functional Assessment Brief, the test of subjective perception of memory, Memory Test Logic I and II and the Action Research Arm Test (ARAT) were used. Results: it was observed that women perceive their memory in a more negative way than men. There was significant correlation between the initial and delayed memory, manual skill on the right side to the left side, manual skill on the right side to the subjective perception of memory. Conclusion: the results of this study support the hypothesis that there is an association between the subjective perception of memory and manual skill. Additional studies on this issue are suggested, once this is an original study.
Key words: Cognition. Geriatrics . Motion. Perception of memory. Manual skill. Digital inclusion. Elderly. Aging.
INTRODUÇÃO

O crescimento da população mundial idosa é um fenômeno que vem ocorrendo de forma significativa. Junto a esse aumento, ganharam notoriedade estudos e pesquisas voltadas para esta clientela, dentro os quais estão os que têm mostrado preocupação com as alterações recorrentes do processo de envelhecimento. ${ }^{1}$

Diversas mudanças ocorrem no processo de envelhecimento - nos seguintes aspectos físicos, psicossociais, funcionais e socioeconômicos. As mudanças físicas são graduais e progressivas e envolvem o declínio da função de todos os órgãos e sistemas corporais de maneira diferenciada; as mudanças psicossociais referem-se às alterações afetivas e cognitivas, relacionando-se aos efeitos fisiológicos do envelhecimento. Nas mudanças funcionais, percebe-se a necessidade de ajuda para desempenhar atividades básicas e por fim, nas mudanças socioeconômicas, a ênfase é dada aos aspectos relacionados à aposentadoria do idoso. ${ }^{2}$

Com relação às mudanças físicas, a mobilidade articular e a força muscular são consideradas importantes quando se fala em habilidades motoras. Estudos mostram que o desempenho motor no processo de envelhecimento tem sido proposto como tendo fator único originário da deterioração da capacidade de processamento central de informação. Para testar essa hipótese, foi realizado um estudo na tentativa de verificar até que ponto poderia se considerar essa visão geral proposta na maioria dos estudos. Os participantes foram submetidos a diversas tarefas motoras. Os resultados mostraram-se contraditórios à hipótese e revelaram que o declínio do desempenho sensório-motor é específico à tarefa e a queda mais acentuada se daria entre os 60 e 70 anos de idade. ${ }^{3}$

É notório que, para realizar satisfatoriamente as tarefas do cotidiano, é preciso destacar a importância da habilidade manual. A mão serve tanto como receptor de informações quanto executor de resposta. As duas funções estão intimamente relacionadas e sofrem influências mútuas. A mobilidade dos dedos tem grande importância para a identificação de objetos. A avaliação funcional da mão pode ser necessária em algumas situações, dentre elas a perda da amplitude de movimento, agilidade e força. A destreza manual também tem sua importância dentro desse contexto. Ela é definida como a capacidade de realizar movimentos habilidosos e dirigidos de braço-mão na manipulação de objetos de maneira controlada. ${ }^{4}$

Um estudo referiu os instrumentos de avaliação funcional que foram analisados quanto aos principais componentes da função manual, são eles: Teste de caixas e blocos, Teste de 
Minnesota de manipulação, Teste de Purdue Pegboard, Teste dos nove pinos, Jebsen Taylor Testeo
Action Research Arm Test (quadro1), todos com aplicabilidade na Terapia Ocupacional. ${ }^{5}$

Quadro 1 - Análise dos instrumentos padronizados de avaliação da função manual. ${ }^{5}$

\begin{tabular}{|l|c|c|c|c|}
\hline \multicolumn{1}{|c|}{ Instrumento padronizado } & $\begin{array}{c}\text { Avalia a } \\
\text { Coordenação } \\
\text { motora grossa }\end{array}$ & $\begin{array}{c}\text { Avalia a } \\
\text { Coordenação } \\
\text { motora fina }\end{array}$ & $\begin{array}{c}\text { Avalia } \\
\text { AVD }\end{array}$ & $\begin{array}{c}\text { Avalia } \\
\text { atividades } \\
\text { bimanuais }\end{array}$ \\
\hline Teste de caixas e blocos & $\mathrm{X}$ & & & \\
\hline Teste de Minnesota de manipulação & $\mathrm{X}$ & $\mathrm{X}$ & & $\mathrm{X}$ \\
\hline Teste de Purdue Pegboard & & $\mathrm{X}$ & & \\
\hline Teste dos nove pinos & & $\mathrm{X}$ & $\mathrm{X}$ & $\mathrm{X}$ \\
\hline Jebsen Taylor Test & & $\mathrm{X}$ & $\mathrm{X}$ & $\mathrm{X}$ \\
\hline Action Research Arm Test & & & & \\
\hline
\end{tabular}

AVD = atividade de vida diária.

Em relação às mudanças cognitivas, a memória vem merecendo destaque. Define-se a memória, portanto, como função mental complexa que leva o indivíduo ao ato de recordar, relembrar e reproduzir o que foi aprendido. Ela envolve a capacidade de registrar, reter e resgatar informações. ${ }^{6}$ Alguns autores sugerem que o declínio intelectual não se encontra dentro do processo normal de envelhecimento, enquanto que outros sugerem que a mente se forma e amadurece junto com o corpo. Entretanto, "há várias evidências de que o desempenho intelectual do idoso apresenta discreta deterioração em tarefas que exigem maior velocidade e flexibilidade no processamento de informações"?

Muitos idosos se queixam de falhas de memória. A forma como o idoso observa sua memória é muito importante para seu desempenho no dia a dia. Trata-se, então, da chamada percepção subjetiva de memória. Vale ressaltar que, quando o idoso verbaliza sobre sua própria memória, provoca em si um momento reflexivo, pois muitas vezes ele não se dá conta de suas dificuldades e este momento pode facilitar a abordagem dos problemas relacionados à memória. ${ }^{6}$

Dentre as queixas relatadas por idosos, existem os chamados "brancos ocasionais", que equivalem ao fato de o idoso não se lembrar onde guardou seus pertences e esquecer o nome de uma pessoa conhecida e que não vê há certo tempo. Com o passar do tempo, os reflexos das "falhas de memória", os "pequenos acúmulos de esquecimento" na vida cotidiana, somados aos efeitos da dinâmica familiar e do grupo de amigos, fazem com que o idoso comece a expressar sentimentos de um "futuro desastroso". 6 Percebe-se, portanto, que a forma como o idoso percebe sua memória é de extrema importância e pode influenciar em diversos aspectos de sua vida cotidiana.

A "explosão tecnológica" que a sociedade vem presenciando tem chamado a atenção para novas discussões e vários aspectos relacionados a esta realidade têm sido envolvidos em estudos. ${ }^{8}$

O processo de inclusão digital (ID) de Idosos já é uma realidade mundial. Uma demonstração disso é a Carta para Inclusão Digital e Social, redigida na França por Ismael Ijji, com a finalidade de promover a ID de homens e mulheres em um contexto social, incluindo cidadãos de todas as idades. Esse documento destaca pontos importantes para que crianças, jovens, adultos e idosos tenham pleno acesso à ID. ${ }^{9}$

Os idosos sentem-se excluídos por não acompanharem a evolução tecnológica que se dá 
de forma cada vez mais rápida e dinâmica. É importante considerar também que as novas tecnologias não atendem às necessidades dessa clientela. Considerando tal realidade, muitos cursos e oficinas foram abertos ao público idoso, fazendo da informática uma realidade na vida de muitos deles.

Com a informática fazendo parte do cotidiano do idoso, muitos aspectos também passaram a ser considerados no que diz respeito a seu desempenho nesses cursos e oficinas. Verificouse, em oficinas realizadas para a inserção do idoso como usuário do computador, que grande parte deles apresenta dificuldades de manuseio do teclado e do mouse, ${ }^{8}$ já que o uso desses acessórios requer uma coordenação motora harmoniosa e no processo de envelhecimento o declínio motor pode manifestar-se. Além deste aspecto, existem outros que estão relacionados ao envelhecimento e que também podem trazer dificuldades no aprendizado do uso do computador: aspectos físicos, visão, audição e declínio no processo cognitivo (como atenção e memória). ${ }^{10}$

Dentro desse processo, observa-se a necessidade de uma equipe multidisciplinar para desenvolver trabalhos direcionados aos idosos. A Terapia Ocupacional tem importante papel nesse contexto, visando à qualidade de vida do idoso (e sua autonomia nas atividades diárias) e a prevenção ou reabilitação do mesmo em seus aspectos cognitivos, físicos, motores, sensoriais e/ou mentais, bem como dificuldade de adaptação ao meio em decorrência dessas disfunções ou de outros processos que venham a desencadear prejuízos à saúde biopsicossocial do indivíduo e da sociedade em que está circunscrito. ${ }^{11}$

Ainda são considerados poucos os estudos voltados para a ID de idosos; entretanto, essa estatística vem se modificando a cada dia. Poucos também são os estudos envolvendo a habilidade manual em idosos.

O objetivo deste estudo foi verificar a associação de memória com habilidade manual em idosos que frequentam as oficinas de inclusão digital (OID) da PUCRS. Trata-se de um estudo original, uma vez que, ao realizar a busca bibliográfica, não se localizou nenhum estudo envolvendo a temática de associação entre percepção de memória, memória lógica inicial e tardia e habilidade manual avaliada através do Action Research Arm Test(ARAT).

As buscas foram realizadas na base de dados do PubMed no período de maio a outubro de 2008, utilizando como descritores perception of memory, manual skill, digital inclusion, elderly e aging.

Assim, este estudo pode trazer contribuições para novas pesquisas e identificar novos conhecimentos, tendo em vista que a ID para idosos é uma realidade ainda recente.

\section{METODOLOGIA}

Trata-se de estudo de corte transversal, descritivo e analítico, com uma amostra de conveniência. A amostra foi composta por 52 idosos do Projeto Potencialldade, oficina de ID de idosos da PUCRS. Os critérios de exclusão $\mathrm{da}$ pesquisa foram não concordar em participar do estudo e apresentar limitações funcionais detectadas a partir da Avaliação Funcional Breve (sendo que apenas um foi excluído, por apresentar limitação motora na Avaliação Funcional Breve - AFB).

A AFB é um instrumento que avalia diversas funções e foi proposto por Lachs et al.. ${ }^{12} \mathrm{O}$ mesmo foi utilizado para retirar do estudo os idosos que possuíssem limitação funcional. A AFB é composta de 11 itens que avaliam áreas específicas como: visão, audição, braço, perna, incontinência urinária, nutrição, estado mental (memória), depressão, atividade de vida diária, ambiente domiciliar e suporte familiar. O teste não possui escore, apenas descreve em cada um dos itens avaliados as características que não devem ser consideradas durante a aplicação do instrumento. Para este estudo, considerou-se apenas a limitação de braços e de memória para cumprimento dos critérios de inclusão. ${ }^{12,13}$

Para obter informações sobre a situação socioeconômica da amostra estudada, foi 
elaborado um questionário geral contendo dados de identificação do idoso, informações socioeconômicas e informações sobre a informática e o uso do computador. Para os propósitos do estudo, foram utilizadas somente a escolaridade e a renda familiar mensal na determinação do perfil socioeconômico da amostra. O instrumento utilizado para avaliar a percepção subjetiva de memória foi o Teste de Percepção Subjetiva de Memória (MAC-Q) proposto por Crook et al. ${ }^{14}$ sendo um instrumento autoadministrado e com objetivo de investigar como o indivíduo percebe sua memória no momento presente, comparandoa quando tinha 40 anos de idade.

A pontuação no teste pode variar de 7 até 35 pontos, sendo a pontuação máxima relacionada com percepção subjetiva maior de disfunção na memória. A versão utilizada neste estudo foi a mesma utilizada pela Escola Paulista de Medicina, onde uma pontuação igual ou maior de 25 pontos é considerada como indicativa de perda subjetiva de memória. ${ }^{15}$

Para avaliar a memória, foram utilizados os Testes de Memória Lógica Ie II, que fazem parte da Escala de Memória de Wechsler. A descrição dos testes consiste na leitura de duas histórias onde a audição tem papel central e, após a leitura, verifica-se o que o indivíduo conseguiu reter de informações. ${ }^{16} J^{1}$ a habilidade manual foi avaliada através do ARAT, que é um instrumento que avalia o desempenho funcional dos membros superiores. É composto por 19 itens e quatro categorias hierárquicas, a saber: agarrar, preensão, pinça e movimentos circulares (coordenação motora grossa). ${ }^{17}$

Os idosos foram informados e convidados a participar do estudo. Foram marcados, com cada um dos idosos, data e horário para uma avaliação inicial (preenchimento dos critérios de exclusão). Posteriormente, foi agendado novo encontro para a aplicação dos demais instrumentos. Para a marcação, levou-se em consideração a participação nas atividades das oficinas de ID para não coincidir com as mesmas.

A aplicação dos instrumentos se deu de forma individual pela pesquisadora principal (terapeuta ocupacional). As avaliações foram realizadas entre outubro e novembro de 2008.

O estudo foi realizado nas dependências do Laboratório de Informática (onde ocorrem as oficinas de ID do Projeto PotencialIdade) e em uma sala de aula da Faculdade de Educação, ambos no $3^{\circ}$ andar do prédio 40 do Campus Central da PUCRS.

As seguintes variáveis foram investigadas: sexo, idade, renda familiar mensal, escolaridade, percepção subjetiva de memória, memória lógica e habilidade manual. Os dados foram previamente testados para normalidade. Os dados com padrão normal foram analisados pela análise de variância Oneway, seguidos por teste $t$ de Student. Os dados com padrão nãonormal foram analisados através da análise de variância não-paramétrica de Kruskeall-Wallis, seguida pelo teste não-paramétrico de Wilcaxon-Mann-Whitney. Os dados categóricos foram analisados pelo teste não-paramétrico do qui-quadrado. No caso de a amostra apresentar uma frequência esperada menor que 05, utilizou-se o teste exato de Fisher.

O estudo foi realizado após apreciação e aprovação da Comissão Científica do IGGPUCRS e do Comitê de Ética em Pesquisa (PUCRS), sob número 1.092/08. Os pesquisadores solicitaram a assinatura do termo de consentimento livre e esclarecido para realização da pesquisa.

\section{RESULTADOS}

Dos 51 idosos avaliados, 10 (19,6\%) eram do sexo masculino e 41 (80,4\%) do feminino, sendo que 23 $(45,1 \%)$ encontravam-se na faixa etária entre 60 e 64 anose $15(29,4 \%)$ na de 70 anos ou mais. A média de idade foi 66,41 $\pm 5,16$ anos. Com relação à escolaridade, $22(43,1 \%)$ possuíam nível superior e nove $(17,7 \%)$ possuíam até o nível médio incompleto. Quanto à renda, 21 (41,2\%) possuíam renda entre dois e cinco salários mínimos. A renda de até um salário mínimo foi observada em três indivíduos (5,9\%).

As características socioeconômicas encontramse descritas na tabela 1. 
Tabela 1 - Distribuição das características demográficas da população estudada (idosos: $n=51$ ). Porto Alegre, RS, 2008.

\begin{tabular}{lc}
\hline \multicolumn{1}{c}{ Variáveis } & $\mathrm{n}(\%)$ \\
\hline Sexo & \\
Masculino & $10(19,6)$ \\
Feminino & $41(80,4)$ \\
Faixa Etária & \\
60-64 anos & $23(45,1)$ \\
$65-69$ anos & $13(25,5)$ \\
$\geqslant 70$ anos & $15(29,4)$ \\
Escolaridade & \\
Ensino fundamental completo ou Ensino Médio incompleto & $09(17,7)$ \\
Médio completo & $20(39,2)$ \\
Superior completo & $22(43,1)$ \\
Renda familiar mensal & \\
Até 1 salário & $03(5,9)$ \\
2-5 salários & $21(41,2)$ \\
6-10 salários & $15(29,4)$ \\
$>10$ salários & $12(23,5)$ \\
\hline
\end{tabular}

Os resultados da análise do desempenho dos idosos na aplicação dos testes propostos no estudo encontram-se nas tabelas 2 e 3 .

A tabela 2 mostra a descrição do desempenho dos idosos na aplicação do Teste de Memória Lógica I, que representa a Memória Inicial (MLI), e do Teste de Memória Lógica II, que representa a Memória Tardia (MLT). Com relação à aplicação do MLI, observou-se que o desempenho dos homens foi similar ao das mulheres. Considerando os resultados obtidos, observa-se que houve desempenho satisfatório dos idosos na aplicação do MLI. O valor de referência do mesmo é de 50 pontos e a média total obtida foi de $18,94 \pm 6,1$.

$\mathrm{Na}$ aplicação do MLT, as médias equiparamse com $25,5 \pm 7,1$ para o sexo masculino e $25,8 \pm 8,11$ para o sexo feminino. A média total foi do MLT foi de 25,7 $\pm 7,8$. Não foram encontrados dados significativos na aplicação do MLI e MLT em relação às demais variáveis do estudo, destacando-se somente certa tendência de associação entre o MLI e a renda $(p=0,070)$. $O$ valor de referência do MLT é de 50 pontos.
Destaca-se então a relação entre MLI e MLT, onde o desempenho dos idosos foi satisfatório nos dois testes.

Em relação à aplicação do Teste de Percepção Subjetiva de Memória (MAC-Q), observou-se que as mulheres têm uma percepção de memória mais negativa do que os homens. A média entre elas foi de $24,07 \pm 4,78$, enquanto que a dos homens foi de $19,9 \pm 6,85$. O resultado do MAC-Q em relação ao sexo foi considerado significativo, com $\mathrm{p}=0,028 \mathrm{na}$ aplicação do Teste T de Student. Em relação às demais variáveis do estudo, nenhum resultado significativo foi encontrado. Percebeuse que, de maneira geral, homens e mulheres apresentaram dificuldades de memória com uma média total de $23,2 \pm 5,4$.

Ressalta-se que o valor máximo de referência do MAC-Q é de 35 pontos e, portanto, quanto maior o valor obtido no teste mais negativo é considerado o resultado. No entanto, só se considera percepção negativa de memória quando o escore final do MAC-Q ultrapassa 25 pontos. Entre 15 e 25 pontos, é possível afirmar que o idoso apresenta dificuldades de memória. 
Levando-se em consideração o sexo, a média do MAC-Q nos homens $(19,9 \pm 6,85)$ foi significativamente menor $(\mathrm{p}=0,028)$ do que nas mulheres $(24,07 \pm 4,78)$.

Tabela 2 - Desempenho nos Testes de Memória Lógica Imediata e Tardia, de acordo com as características demográficas (idosos: $\mathrm{n}=51$ ). Porto Alegre, RS, 2008.

\begin{tabular}{|c|c|c|c|c|}
\hline & $\begin{array}{c}\text { MLI } \\
\mathrm{m} \pm \mathrm{dp}\end{array}$ & $P$ & $\begin{array}{c}\text { MLT } \\
\mathrm{m} \pm \mathrm{dp}\end{array}$ & $P$ \\
\hline Sexo & & 0,510 & & 0,907 \\
\hline Masculino & $20,1 \pm 7,2$ & & $25,5 \pm 7,1$ & \\
\hline Feminino & $18,6 \pm 5,8$ & & $25,8 \pm 8,1$ & \\
\hline Faixa Etária & & 0,863 & & 0,844 \\
\hline 60-64 anos & $18,4 \pm 6,9$ & & $25,0 \pm 10,1$ & \\
\hline 65-69 anos & $19,0 \pm 26,3$ & & $26,3 \pm 4,5$ & \\
\hline$\geqslant 70$ anos & $19,6 \pm 6,9$ & & $26,3 \pm 6,2$ & \\
\hline Escolaridade & & 0,114 & & 0,091 \\
\hline Até Médio incompleto & $17,1 \pm 6,1$ & & $24,0 \pm 7,6$ & \\
\hline Médio completo & $21,1 \pm 6,2$ & & $28,7 \pm 6,4$ & \\
\hline Superior completo & $17,6 \pm 5,5$ & & $23,7 \pm 8,5$ & \\
\hline Renda & & 0,070 & & 0,100 \\
\hline Até 1 salário & $11,0 \pm 2,6$ & & $15,0 \pm 11,3$ & \\
\hline 2-5 salários & $18,1 \pm 6,0$ & & $26,0 \pm 9,1$ & \\
\hline 6-10 salários & $20,3 \pm 6,5$ & & $26,4 \pm 4,5$ & \\
\hline > 10 salários & $20,5 \pm 4,8$ & & $27,2 \pm 6,5$ & \\
\hline TOTAL & $18,9 \pm 6,1$ & & $25,7 \pm 7,8$ & \\
\hline
\end{tabular}

MLI = Memória Lógica Inicial; MLT = Memória Lógica Tardia; $P=$ nível de significância do teste.

A tabela 3 mostra a descrição do desempenho dos idosos na aplicação do ARAT, que é o teste de investigação da ação do braço. O teste avalia o lado direito (ARAT-D) e o lado esquerdo (ARAT-E). Com relação à aplicação do ARAT-D e do ARAT$E$, observou-se que homens e mulheres praticamente mantiveram as mesmas médias de desempenho, com $53,80 \pm 1,619$ para o lado direito dos participantes do sexo masculino e 53,41 $\pm 2,249$ para os participantes do sexo feminino. No lado esquerdo, a média entre os homens foi de $53 \pm 2,944$ e das mulheres, $53,54 \pm 2,550$. A média total para ambos os lados foi de 53,4 $\pm 2,3$ para o ARAT-D e $53,4 \pm 2,6$ para o ARAT-E.

Não foram encontrados dados significativos com relação ao ARAT-D e ARAT-E e as demais variáveis do estudo, tendo todos os resultados apresentado $\mathrm{p}>0,050$. Os resultados permitiram observar que os idosos se saíram bem no teste em ambos os lados, considerando que o valor de referência total do ARAT é de 57 pontos. 
Tabela 3 - Desempenho no Action Research Arm Test (ARAT), de acordo com as características demográficas (idosos: $\mathrm{n}=51$ ). Porto Alegre, RS, 2008.

\begin{tabular}{|c|c|c|c|c|}
\hline Variáveis & $\begin{array}{l}\text { ARAT-D } \\
m \pm d p\end{array}$ & $\mathrm{p}$ & $\begin{array}{l}\text { ARAT-E } \\
\mathrm{m} \pm \mathrm{dp}\end{array}$ & $\mathrm{p}$ \\
\hline Sexo & & 0,652 & & 0,565 \\
\hline Masculino & $53,8 \pm 1,6$ & & $53 \pm 2,9$ & \\
\hline Feminino & $53,4 \pm 2,2$ & & $53,5 \pm 2,5$ & \\
\hline Faixa Etária & & 0,626 & & 0,405 \\
\hline 60-64 anos & $53,1 \pm 2,6$ & & $53,1 \pm 2,6$ & \\
\hline 65-69 anos & $53,8 \pm 2,2$ & & $53,0 \pm 2,8$ & \\
\hline$\geqslant 70$ anos & $53,7 \pm 2,1$ & & $54,2 \pm 2,3$ & \\
\hline Escolaridade & & 0,204 & & 0,212 \\
\hline Até Médio incompleto & $54,7 \pm 1,9$ & & $54,4 \pm 2,6$ & \\
\hline Médio completo & $53,1 \pm 1,8$ & & $52,7 \pm 2,3$ & \\
\hline Superior completo & $53,2 \pm 2,8$ & & $53,6 \pm 2,6$ & \\
\hline Renda Familiar Mensal & & 0,942 & & 0,608 \\
\hline Até 1 salário & $53,6 \pm 3,2$ & & $53 \pm 3$ & \\
\hline 2-5 salários & $53,3 \pm 2,4$ & & $53,1 \pm 2,5$ & \\
\hline 6-10 salários & $53,8 \pm 2,7$ & & $54,2 \pm 2,9$ & \\
\hline > 10 salários & $53,3 \pm 1,6$ & & $53 \pm 2,3$ & \\
\hline TOTAL & $53,4 \pm 2,3$ & & $53,4 \pm 2,6$ & \\
\hline
\end{tabular}

ARAT-D = Investigação da Ação do Braço Direito; ARAT-E = Investigação da Ação do Braço Esquerdo.

Os resultados da análise de correlação entre o MLI, MLT, MAC-Q, ARAT-D e ARAT-E encontram-se na tabela 4.

Na correlação entre o MLI, MAC-Q, ARATD e ARAT-E, observou-se que os dados não foram significativos, ou seja, não houve correlação relevante entre eles. Entretanto, houve correlação entre o MLI e o MLT, com $\mathrm{r}=0,76$ e $\mathrm{p}<0,001$. Este resultado demonstra que a memória imediata está diretamente relacionada com a memória tardia e se uma não se sai bem, a outra também não obtém desempenho satisfatório. Não houve diferenças significativas entre o MLT, MAC-Q, ARAT-D e ARAT-E. Observou-se que houve somente correlação entre MLT e MLI, que apresentam os mesmos resultados citados anteriormente $(\mathrm{r}=0,76$ e $\mathrm{p}<0,001)$.
No que diz respeito à correlação entre o ARATD, MAC-Q, MLI, MLT, ARAT-E, só houve resultados significativos entre o ARAT-D e o MAC$\mathrm{Q}$ com $\mathrm{r}=0,29$ e $\mathrm{p}=0,036$. Esses resultados demonstram que a percepção subjetiva de memória do idoso tem grande importância para a habilidade manual do lado direito, considerando que a maioria dos idosos participantes são destros e, portanto, utilizam o mouse com o referido lado nas OID, o que pode influenciar na qualidade de manuseio do referido acessório.

Na correlação entre ARAT-E, MAC-Q, MLI, MLT e ARAT-D, observou-se que houve resultados significativos somente entre o ARAT-E e ARAT$\mathrm{D}$, com $\mathrm{r}=0,79$ e $\mathrm{p}<0,001$. Tal resultado demonstra que a habilidade manual tem grande importância para o desempenho dos idosos. 
Tabela 4 - Coeficientes de correlação de Pearson e valores de P, entre os Testes de Memória Lógica Inicial, Memória Lógica Tardia, Percepção Subjetiva de Memória e Teste motor (idosos: $\mathrm{n}=51$ ). Porto Alegre, RS, 2008.

\begin{tabular}{lcccc}
\hline & MAC-Q & MLI & MLT & ARAT-D \\
\hline MLI & $\mathrm{r}=0,14$ & & & \\
& $\mathrm{p}=0,921$ & & & \\
MLT & $\mathrm{r}=-0,48$ & $\mathrm{r}=0,76$ & & \\
& $\mathrm{p}=0,737$ & $\mathrm{p}<0,001$ & & \\
ARAT-D & $\mathrm{r}=-0,29$ & $\mathrm{r}=0,080$ & $\mathrm{r}=0,94$ & \\
& $\mathrm{p}=0,036$ & $\mathrm{p}=0,577$ & $\mathrm{p}=0,513$ & \\
ARAT-E & $\mathrm{r}=-0,08$ & $\mathrm{r}=0,16$ & $\mathrm{r}=0,12$ & $\mathrm{r}=0,79$ \\
& $\mathrm{p}=0,558$ & $\mathrm{p}=0,246$ & $\mathrm{p}=0,394$ & $\mathrm{p}<0,001$ \\
\hline
\end{tabular}

MLI = Memória Lógica Inicial; MLT = Memória Lógica Tardia; MAC-Q = Percepção Subjetiva de Memória; ARAT-D = Investigação da Ação do Braço Direito; ARAT-E= Investigação da Ação do Braço Esquerdo.

\section{DISCUSSÃO}

O presente estudo avaliou a habilidade manual, a percepção subjetiva de memória e a memória lógica, e investigou a associação entre a habilidade manual e a percepção subjetiva de memória.

Foram localizados apenas seis estudos internacionais envolvendo motricidade e aspectos cognitivos, um dos quais foi publicado em japonês. ${ }^{18}$ A seguir, serão apresentados alguns aspectos relevantes desses estudos.

Smith et al. ${ }^{19}$ avaliaram 497 adultos saudáveis entre 18 e 94 anos de idade, utilizando testes para avaliar a idade e aprendizagem, lembrança e execução de tarefas visuomotoras. Os indivíduos com idade superior a 58 anos foram recrutados como grupo controle de um estudo longitudinal de pacientes com doença de Alzheimer. O estudo concluiu que o tempo de execução das tarefas diminui com a idade, e no estudo longitudinal a aprendizagem motora foi mais lenta a partir de 62 anos de idade e a memória motora foi preservada entre os idosos com idade entre $18 \mathrm{e}$ 94 anos. O estudo ainda ressalta que não há conhecimento sobre a duração máxima de uma memória motora.
Outra pesquisa encontrada foi realizada por Voelcker-Rehage \& Alberts, ${ }^{20}$ e teve como objetivo determinar os efeitos da prática motora no desempenho cognitivo e motor dos idosos em condições simples e em duplas tarefas. Participaram do estudo 14 jovens com idades entre 19 a 28 anos e 12 idosos com idades entre 67 e 75 anos. Os participantes passaram por um pré-teste e um pós-teste da prática motora e também por tarefas cognitivas. Os resultados apontaram que, nas tarefas cognitivas, houve melhor desempenho dos jovens em relação aos idosos, porém nas tarefas motoras, ambos os grupos apresentaram melhora em relação à força em condições simples e duplas tarefas.

O estudo de Miyahara ${ }^{21}$ sobre a memória de trabalho e o movimento das mãos mostrou um declínio relativo à idade para recrutar e reter espontaneamente uma estratégia verbal para lembrar os movimentos das mãos.

O estudo de Boyd et al..$^{22}$ teve como objetivo identificar características envolvendo os aspectos motores, espaciais e temporais que pudessem ser marcantes na sequência de aprendizagem motora. Buscou também verificar se a idade afeta diretamente essa aprendizagem, mostrando que nos jovens e adultos de meia-idade, o aprendizado 
das sequências múltiplas do movimento tem como elemento mais importante o aprendizado motor; nos idosos mais velhos não houve mudança da sequência em tal aprendizado, sugerindo um declínio devido à idade mais avançada.

Por fim, o estudo exploratório de Stoter et al. ${ }^{23}$ comparou a sequência de aprendizagem motora em adultos jovens com idade média de 25 anos e idosos com idade média de 63 anos. Mostrou em seus resultados que a precisão dos idosos foi menor que a dos adultos jovens, embora a capacidade de memória tenha sido equivalente em ambos os grupos. O referido estudo aponta ainda a importância do cuidado na avaliação desses resultados, considerando que a amostra foi relativamente pequena (15 participantes por grupo etário).

Os estudos anteriormente mencionados revelam resultados de certa forma semelhantes entre si. Porém, não envolvem a temática de associação entre percepção de memória, memória lógica inicial e tardia e habilidade manual avaliada através do ARAT, envolvida neste estudo.

Para avaliar a memória dos idosos, foram utilizados o Teste de Memória Lógica I e II, que fazem parte da Escala de Wechsler. Estudo realizado por Nitrini et al., ${ }^{24}$ com o objetivo de recomendar condutas baseadas em evidências para diagnosticar a doença de Alzheimer, revelou que a escala de Wechsler encontra-se dentre as mais eficazes na avaliação de memória. Na população idosa, a Escala de Wechsler é de grande valia não por avaliar o quoeficiente intelectual, mas por avaliar funções cognitivas específicas em cada subteste que compõe a escala. ${ }^{25}$

Outra pesquisa realizada por Quadros Júnior et al. ${ }^{26}$ também avaliou a memória de idosos institucionalizados utilizando uma análise por gênero. Os testes de Memória Lógica I e II foram os instrumentos utilizados. $\mathrm{O}$ resultado do desempenho dos idosos nos testes de memória aplicados no estudo foi considerado altamente prejudicado, mas tal resultado já era de se esperar, em virtude do comprometimento cognitivo encontrado na população estudada.
O desempenho dos idosos participantes do Projeto PotencialIdade nos Testes de Memória Lógica I e II foi maior que os encontrados nos estudos anteriormente citados. Obviamente, é importante considerar as diferenças entre as populações estudadas, mas se pode também observar a importância de o idoso manter-se ativo. As oficinas de ID, nesse sentido, permitem ao idoso buscar novos conhecimentos, desenvolver e/ou manter habilidades.

Autores como Xavier et al. ${ }^{27}$ e Wantanabe ${ }^{28}$ confirmam tal pensamento e reforçam que a ID promove a capacidade de pensar, propõe melhorias nos aspectos motores, percepto-cognitivos, Atividades de Vida Diária (AVDs) e Atividades Instrumentais de Vida Diária (AIVDs), aspectos estes trabalhados pelo terapeuta ocupacional em sua intervenção. ${ }^{28,29}$

Em relação à aplicação do MAC-Q em estudos brasileiros, Xavier et al. ${ }^{30}$ utilizaram esse teste em pesquisa com 66 idosos, comparando-o com testes específicos de memória, com o objetivo de examinar se a percepção de perda de memória era um índice acurado de real distúrbio de memória. A conclusão a que os autores chegaram foi de que, apesar de isoladamente o MAC-Q conter um valor preditivo para futura demenciação, a opinião do idoso sobre sua memória e a testagem específica da memória não estão correlacionadas.

Outro estudo, realizado por Yassuda, ${ }^{31}$ utilizou o MAC-Q para avaliar a metamemória em ensaio clínico com 69 idosos considerados saudáveis. Verificou-se, nos resultados, que houve menor número de queixas de memória no pósteste tanto no grupo experimental quanto no grupo controle.

No estudo realizado por Guerreiro et al., ${ }^{32}$ os autores estudaram as características de idosos que buscam treinamento de memória na Universidade Aberta da Terceira Idade do Rio de Janeiro e identificaram possíveis fatores preditores de prejuízos cognitivos. A média do desempenho dos idosos na aplicação do MAC-Q foi de $23,22 \pm 4,06$, estando inversamente relacionado com a escolaridade. 
Os idosos do Projeto Potencialldade apresentaram resultados semelhantes no MAC-Q $(23,2 \pm 5,4)$, mas não houve correlação da escolaridade com o MAC-Q. Comparando esses resultados observa-se que, apesar de o nível de escolaridade dos idosos participantes do Projeto PotencialIdade ser alto (a maioria possui nível superior completo), as queixas de memória não diminuíram, conforme o resultado exposto no estudo de Guerreiro et al. ${ }^{32}$ Mesmo considerando as diferenças entre os estudos, nota-se que a percepção de memória dos idosos é de extrema importância quando os mesmos se propõe a participar de algum tipo de atividade.

Outros estudos também avaliaram a percepção de memória em idosos de Porto Alegre. Como exemplo, tem-se o estudo cujo objetivo era determinar e comparar o desempenho no MiniExame do Estado Mental (MEEM) em idosos da comunidade de Porto Alegre e de VeranópolisRS. A média do MAC-Q para os idosos de Porto Alegre foi de 25,87 \pm 4,93 e dos idosos de Veranópolis foi de $26,70 \pm 4,71$. No resultado do perfil sociodemográfico do estudo, verificou-se que a maioria dos idosos encontrava-se na faixa etária de 60 e 69 anos (48,8\%) e com segundo grau completo (ou acima), representando 41,5\% do total de participantes do estudo. ${ }^{33}$

Os resultados do referido estudo não se enquadram nos resultados obtidos nesta pesquisa. Os participantes tanto de Porto Alegre quanto de Veranópolis apresentaram percepção negativa de memória (MAC - $\mathrm{Q}>25$ ). O nível de escolaridade em ambos os estudos é satisfatório, mas mesmo assim não modificou a maneira como os idosos percebem sua memória. Estes resultados se confrontam com o estudo de Guerreiro et al., ${ }^{32}$ que mostrou resultados contrários.

É importante mencionar que há diferenças nas características da população estudada. O estudo de Nascimento ${ }^{33}$ constituiu-se de idosos com e sem comprometimento cognitivo, os participantes do estudo de Guerreiro et al..$^{32}$ foram alunos que se interessaram em participar de oficinas de memória da UERJ, sendo excluídos aqueles participantes que eram analfabetos e os que apresentaram existência de doença psiquiátrica e síndrome demencial em estágio intermediário ou avançado. A população estudada neste trabalho constituiu-se de idosos que participam de oficinas de ID e, portanto, sem comprometimentos cognitivos.

Como mencionado anteriormente, o próprio idoso pode verbalizar o que acha sobre sua memória, provocando assim um momento reflexivo sobre o mesmo, pois muitas vezes o idoso não se dá conta de suas dificuldades e esse momento pode facilitar a abordagem dos problemas relacionados à memória. ${ }^{6}$

Considerando o pensamento de Vieira, ${ }^{6}$ percebeu-se que nesse estudo a maior dificuldade dos idosos está na sua autoconfiança, pois em sua percepção de memória as queixas não se apresentam como uma realidade quando comparadas aos resultados dos testes de Memória Lógica. Portanto, o momento reflexivo referido por Vieira ${ }^{6}$ permite ao idoso perceber que pode ter um desempenho de memória superior ao que imagina e que essa percepção diferenciada é o que vai impulsioná-lo a novas maneiras de lidar com sua memória, contribuindo assim na formulação de novas percepções, dessa vez, mais positivas.

A habilidade manual dos idosos foi avaliada através do Action Research Arm Test (ARAT). A maioria dos estudos encontrados envolvendo o uso deste instrumento durante o levantamento bibliográfico avaliava idosos que já possuíam algum comprometimento motor; geralmente decorrente de um AVC (acidente vascular cerebral), tais como os estudos de Yozbatiran, ${ }^{34}$ Page $^{35}$ e Van der Lee. ${ }^{36}$

O uso do ARAT no Brasil ainda é pouco frequente. A maioria dos estudos encontrados eram publicações estrangeiras. O ARAT (também pode ser usada a sigla ARA - Action Research Arm) foi elaborado em 1981 por Lyle para avaliar a função da extremidade superior. ${ }^{34,36} \mathrm{~A}$ confiabilidade do ARAT foi positiva em todos os estudos pesquisados, apesar de ainda haver alguns questionamentos referentes ao mesmo. 
Pode-se observar tal confiabilidade nos estudos de Yozbatiran, ${ }^{34}$ Page ${ }^{35} \mathrm{e}$ Van der Lee. ${ }^{36}$ Sua escolha para este estudo teve a intenção de verificar como seria usá-lo em uma população diferenciada com máxima independência no desempenho de suas tarefas e sem comprometimentos motores. Durante a pesquisa, procurou-se um ponto de corte para o ARAT. Este poderia determinar até qual pontuação específica poderia se avaliar a habilidade motora de maneira positiva ou negativa. Entretanto, não foram encontrados estudos que mencionassem tal informação. Os achados obtidos referiam-se geralmente a comparações entre o ARAT e outros instrumentos, e também análises do mesmo. De acordo com o estudo de Yozbatiran, ${ }^{34}$ quanto mais alto for o escore total no ARAT, melhor é o status motor do braço. Essa referência foi utilizada como base para avaliação da habilidade manual do estudo aqui apresentado.

A média geral do desempenho dos idosos do Projeto PotencialIdade na aplicação do ARAT, envolvendo ambos os sexos, foi de $54,7 \pm 1,9$ para o lado direito e de 54,4 $\pm 2,6$ para o lado esquerdo. Considerando que o valor de referência é de 57 pontos e considerando também o estudo de Yozbatiran, ${ }^{34}$ observa-se que os idosos apresentaram ótima habilidade manual. É interessante observar nesses resultados que tanto o desempenho do lado direito quanto o do lado esquerdo dos idosos foi alto, havendo boa correlação entre o ARAT-D e ARAT-E $(r=0,79$; $\mathrm{p}<0,001)$.

Spirduso, ${ }^{37}$ em sua literatura, aponta que a questão levantada sobre os efeitos do envelhecimento nas habilidades de múltiplos membros é se a preferência por uma das mãos pode ser atenuada nos idosos. As crianças desenvolvem uma preferência por uma das mãos no seu crescimento e desenvolvimento, e pode-se especular, portanto, que conforme as pessoas envelhecem podem tornar-se de certa forma ambidestras. As habilidades funcionais envolvem atividades do dia a dia que utilizam a coordenação de dois ou mais movimentos de uma só vez. Essas tarefas incluem digitar num escritório, usar garfo e faca, discar o número de um telefone, dentre outros que, com o processo de envelhecimento, podem trazer dificuldades na coordenação dessas tarefas para alguns indivíduos. As habilidades funcionais, portanto, envolvem dois tipos de coordenação importantes: as motoras finas (como por exemplo, digitar, selecionar cartas, discar um número telefônico) e motoras grossas (como por exemplo, varrer, limpar com esfregão, arremessar).

Houve correlação significativa entre MLI e MLT, com $r=0,76$ e $p<0,001$. Os resultados indicam que se a Memória Imediata vai bem, a tardia também e vice-versa. A Memória Imediata refere-se à Memória de Curta Duração (Memória de Trabalho) e se estende desde os primeiros segundos ou minutos após o aprendizado até três a seis horas, tempo que a memória de longa duração leva para ser consolidada, ou seja, construída. Já a Memória Tardia refere-se à Memória de Longo prazo, que é responsável pelo armazenamento da informação por um período longo de tempo (horas ou semanas). ${ }^{38}$

Estudo realizado por Parente et al. ${ }^{39}$ revela que recontar histórias é uma atividade complexa que envolve a Memória de Curta Duração e a Memória Episódica. O referido estudo buscou verificar, numa população brasileira, se no desempenho de jovens e adultos com mais idade há uma possível interação entre o tipo de estrutura memorizada, as estratégias distintas de memorização e a idade. Trinta e um sujeitos ouviram e recontaram uma história contada pelo examinador; 17 pessoas formaram o grupo de adultos jovens com idade entre 30 e 55 anos (grupo adulto) e 14 o grupo de adultos mais velhos, com mais de 60 anos (grupo idoso). Cada sujeito foi instruído a ouvir atentamente a história e depois recontá-la com o maior número de detalhes possível. Os resultados apontaram que os adultos mais jovens lembraram mais a macroestrutura do texto do que os idosos, mas ambos os grupos relembraram melhor as macroestruturas do que as microestruturas, e não foi encontrada diferença no número de inferências (comentários pertinentes a respeito de fatos presentes na história original), interferências (quando o sujeito modifica o significado das proposições da história) e reconstruções (a introdução de proposições que 
relatam fatos não presentes na história original). Houve também um desempenho menor na Memória de Curto Prazo.

Os resultados desse estudo de certa forma se assemelham aos resultados obtidos na correlação entre MLI e MLT. A maioria dos idosos se saiu melhor no momento de recontar a história pela segunda vez e também lembraram mais da macroestrutura da história; entretanto, observou-se que não houve diferenças significativas em relação a comentários pertinentes de fatos presentes na história original, modificações na estrutura da história contada e introdução de informações não contidas na história original. Observou que o bom funcionamento da Memória Inicial contribui para o bom funcionamento da Memória Tardia. Esta correlação vai ao encontro do processamento da memória, que envolve a atenção e recepção da informação, o armazenamento da informação e a recuperação ou resgate da informação..$^{40} E^{\prime}$ nesse processo de memorização que se desenvolvem os tipos de memória, desde a de curta duração até as demais. ${ }^{38}$

Foi observada correlação negativa fraca entre o ARAT-D e MAC-Q ( $\mathrm{r}=-0,29$ e $\mathrm{p}=0,036)$. Observa-se, através desses resultados, que quanto mais alto for o desempenho no teste motor, melhor é a percepção subjetiva de memória, considerando que o escore diminuiria neste teste. Não foi encontrado nenhum artigo que tenha estudado a percepção de memória e a habilidade manual em idosos participantes de OID, nem mesmo estudos envolvendo somente a percepção de memória e a habilidade manual em idosos. Trata-se, portanto, de uma descrição inédita que levanta alguns questionamentos e proporciona um grande campo de investigação.

Apesar da correlação do ARAT-D com a percepção subjetiva da memória, não foi verificada qualquer associação do mesmo com a avaliação objetiva nos testes de MLI e MLT. Isto pode sugerir que a habilidade manual esteja relacionada mais à memória semântica e não à memória episódica, declarativa, sendo a deficiência da primeira mais percebida pelo idoso na avaliação do MAC-Q. Isto também explicaria a nãoassociação entre as avaliações subjetiva (MAC-Q) e objetiva (MLI e MLT). Adicionalmente, a associação apenas com o lado direito pode decorrer do fato da maioria dos indivíduos terem dominância manual direita e, portanto, apresentarem um comprometimento mais perceptivo da habilidade manual relacionado com a pior percepção da memória. Estudos adicionais são necessários para esclarecimento dessas questões.

\section{CONCLUSÃO}

Os resultados obtidos no presente estudo mostram que idosos participantes de uma oficina de inclusão digital apresentam um resultado satisfatório nas avaliações subjetivas e objetivas de memória e de habilidade manual. Foi observada forte correlação entre a memória imediata e a tardia, não havendo associação destas com a percepção subjetiva de memória. A habilidade manual não mostrou nenhuma correlação com a avaliação objetiva da memória, mas foi observada correlação negativa fraca, estatisticamente significativa, entre o MAC-Q (que mede a percepção subjetiva da memória, sendo o maior valor relacionado com uma memória percebida como pior) e o ARAT-D (que mede a habilidade manual do lado direito). Este resultado sugere que possa haver uma possível associação da habilidade manual, principalmente do lado direito, com a memória semântica e não episódica, e que alterações da primeira possam ser mais percebidas pelo MACQ. Contudo, estudos adicionais são necessários para esclarecer essas questões.

\section{AGRADECIMENTOS}

Os autores agradecem aos idosos participantes do Projeto Potencialldade, bem como aos professores das oficinas (Anderson Jackle, Ângela Fraquelli, Cláudia Tacques e Letícia Machado), pelo auxílio na coleta dos dados e apoio constante durante a execução deste estudo. 


\section{REFERENCIAS}

1. Cunha NV. The challenge of an Anthropology of the ages. Hist Ciênci saúde-Manguinhos 2000; 6(3): $:-14$

2. França LS. Quando o entardecer chega : o envelhecimento ainda surpreende muitos [ Acesso em 6 mai 2003]. Disponível em: URL: http://www.guiarh.com.br/pp46.html.

3. Teixeira LA. Declínio de desempenho motor no envelhecimento é específico à tarefa. Rev Bras Med Esport 2006 dez; 12(6): 351-55.

4. Desai $\mathrm{K}$,et al. Normative data of purdue pegboard on indian population. IJOT 2006; 37(3): 69-72.

5. Rodrigues AS, et al. Instrumentos padronizados de avaliação da função manual- revisão de literatura e análise crítica da aplicabilidade na terapia Ocupacional. Rev Cient Faminas 2007 Jan; 3(1): 54.

6. Vieira EB. Manual de gerontologia: um guia teórico-prático para profissionais, cuidadores e familiares. Rio de Janeiro: Revinter; 1996. 187 p.

7. Almeida OP. Queixa de problemas com a memória e o diagnóstico de demência. Arq Neuro-psiquiatr 1998; 56(3) : 605-612

8. Raabe et ,al. Promovendo a inclusão digital dos idosos através de práticas de design participatório. Contrapontos $2005 \mathrm{dez}$; 5(3): 417-30.

9. Ijji Ismael. Charte pour l'inclusion numérique et sociale; 2003 [ Acesso em 3 mai 2003].Disponível em: URL: http:// www.tanmia.ma/article.php3?id_article $=4965$.

10. Bean C. Meeting the challenge: training an aging population to use computers. Southeastern Librarian 2003 out; 51(3):16-25.

11. Profala. A atuação as terapia ocupacional junto ao idoso [ Acesso em 10 mar 2010].

Disponível em: URL: http:// www.profala.com/artto9.htm.

12. Lachs MS ,et al. A simple procedure for general screening for functional disability in elderly patients. Ann Intern Med 1990; 112(9): 699-706.

13. Moore AA,et al. A Randomized trial of officebased screening for common problems in older persons. Am J Med 1997; 102(4): 371-8.

14. Crook TH, et al. Assessment of memory complaints. In: Age-associated memory impairment. The MAC-Q. International Psychogeriatry 1992; 4(2): 165-76.
15. Bertolucci PH, et al. The Mini-Mental state examination in a general population: impact of educational status. Arq. Neuropsiquiatr 1994; 52 (1): 1-7.

16. Wechsler D. WMS-R : manual. Toronto: The Psychological Corporation Harcout Brace Jovanovich; 1987.

17. Van Der Lee JH, et al. Improving the action research arm test: a unidimensional hierarchical scale. Clinical Rehabilitation 2002; 16(6): 646-53.

18. Chiba $S$, et al. Effects of aging on hand coordination: a quantitative analysis of ability to track moving targets. Nippon Ronen Igakkai Zasshi 1987; 24(2): $132-7$.

19. Smith $\mathrm{CD}$, et al. Memories that last in old age: motor skill learning and memory preservation. Neurobiol Aging 2005; 26(6): 883-90.

20. Voelcker-Rehage C, Alberts JL. Effect of motor pratice on dual-task performance in older adults. J Gerontol 2007; 62(3): 141-8.

21. Miyahara M. Aging Influences on work memory for hand movements: a test of the metamemory deficit hypothesis. Experimental aging rasearch 2007; 33(4): 417-28.

22. Boyd LA, et al. Multidimensional motor sequence learning is impaired in older but not younger or middle-aged adults. Physical Therapy 2008; 88(3): 351-62.

23. Stoter AJ, et al. Rehearsal strategies during motor-sequence learning in old age: execution vs motor imagery. Perceptual and motor skills 2008; 106(3): 967-78.

24. Nitrini R, et al. Diagnóstico da doença de alzheimer no Brasil: avaliação cognitiva e funcional. Arq Neuro-psiquiatr 2005; 63(3): 713-719

25. Azambuja LS. Avaliação neuropsicológica do Idoso. RBCEH 2007 dez.; 4(2): 40-45.

26. Quadros Júnior AC, et al. Estudo do nível de atividade física, independência funcional e estado cognitivo de Idosos institucionalizados: análise por gênero. Brazilian Journal of Biomotricity 2008; 2(1): 39-50.

27. Xavier A, et al. Oficina da lembrança: ambiente de inclusão digital e Tele-reabilitação cognitiva para Idosos, portadores de doença de Alzheimer e outros distúrbios cognitivos [Acesso em 12 mai 2009].Disponível em: URL: http:// telemedicina.unifesp.br/pub/SBIS/CBIS2004/ trabalhos/arquivos/782.pdf. 
28. Watanabe M, et al. Terapia Ocupacional e o uso do computador como recurso terapêutico. Acta Fisiátrica 2003 Abr; 10(1): 17-20.

29. Lindôso ZCL. Acompanhamento terapêutico ocupacional nas oficinas de Inclusão Digital. In: Ferreira AJ ,et al (Orgs). Inclusão digital de idosos: a descoberta de um novo mundo. Porto Alegre: EDIPUCRS; 2008. p. 59-73.

30. Xavier FMF, et al. A relevância clínica da percepção subjetiva de perda de memória por pessoas de idade. Rev Bras Neurol 2001; 37(1).

31. Yassuda MS, et al. Treino de memória no idoso saudável: benefícios e mecanismos. Psicologia: reflexão e crítica 2006; 19(3): 470-481.

32. Guerreiro TC,et al. Queixa de memória e disfunção objetiva de memória em idosos que ingressam na oficina da memória na UnATI/ UERJ. Rev Bras Geriatr Gerontol 2006; 9(1) : 720.

33. Nascimento NMR. Estudo comparativo sobre a prevalência de declínio cognitivo entre dois grupos de Idosos .Porto Alegre.Dissertação [ Mestrado em saúde pública] -Pontifícia
Universidade Católica do Rio Grande do Sul; 2008.

34. Yozbatiran $\mathrm{N}$, et al. A standardized pproach to performing the action research arm test. NNR 2008; 22(1):78-90.

35. Page SJ, Levine P, Leonard A. Mental practice in chronic stroke: results of a randomized, placebocontrolled trial. Stroke 2007; 38: 1293-97.

36. Van Der Lee JH et al. The intra-and interrater reability of the Action research arm test: a pratical test of upper extremity function in patients with stroke. Archives of Physical Medicine Rehabilitation 2001; 82: 14-19.

37. Spirduso WW. Dimensões físicas do envelhecimento. Barueri: Manole; 2005.

38. Izquierdo I. Memória. Porto Alegre: Artmed; 2002. p. 95.

39. Parente MAMP, et al. Ativação de modelos mentais no recontar de histórias por idosos. Psicologia: reflexão e crítica 1999; 12(1) : 157-172

40. Alvarez A. Exercite sua memória. São Paulo: Nova Cultural; 2004. 